\title{
Correlation of Gastrointestinal Symptoms at Initial Presentation with Clinical Outcomes in Hospitalized COVID-19 Patients: Results from a Large Health System in the Southern USA
}

\author{
Harsh K. Patel ${ }^{1}$ D . Rosemary Kovacic ${ }^{2} \cdot$ Viveksandeep Thoguluva Chandrasekar $^{3} \cdot$ Sneha C. Patel ${ }^{4} \cdot$ Munraj Singh $^{5}$. \\ Elise Le Cam ${ }^{5}$. Jeffrey H. Burton ${ }^{6} \cdot$ Arnab Ray $^{2} \cdot$ Janak N. Shah ${ }^{2}$
}

Received: 22 May 2021 / Accepted: 8 January 2022 / Published online: 7 February 2022

(c) The Author(s), under exclusive licence to Springer Science+Business Media, LLC, part of Springer Nature 2022

\begin{abstract}
Background We aimed to understand the association of gastrointestinal (GI) symptoms at initial presentation with clinical outcomes during COVID-19 hospitalization.

Methods This retrospective, multicenter cohort study included consecutive hospitalized COVID-19 patients from a single, large health system. The presence of GI symptoms was assessed at initial presentation and included one or more of the following: nausea, vomiting, diarrhea and abdominal pain. Patients were divided into three cohorts: Only GI symptoms, GI and non-GI symptoms and only non-GI symptoms. The primary outcome was association of GI symptoms with mortality. Secondary outcomes included prevalence of GI symptoms and survival analysis.

Results A total of 1672 COVID-19 patients were hospitalized (mean age: $63 \pm 15.8$ years, females: $50.4 \%$ ) in our system during the study period. $40.7 \%$ patients had at least one GI symptom (diarrhea in $28.3 \%$, nausea/vomiting in $23 \%$, and abdominal pain in $8.8 \%$ patients), and $2.6 \%$ patients had only GI symptoms at initial presentation. Patients presenting with GI symptoms (with or without non-GI symptoms) had a lower mortality rate compared to patients presenting with only nonGI symptoms ( $20 \%$ vs. $26 \% ; p<0.05)$. The time from hospitalization to being discharged was less for patients presenting with only GI symptoms ( 7.4 days vs. $>9$ days, $p<0.0014$ ). After adjusting for other factors, the presence of GI symptoms was not associated with mortality $(p>0.05)$.

Conclusion Among a hospitalized COVID-19 positive Southern US population, 41\% patients presented with either diarrhea, nausea, vomiting or abdominal pain initially. The presence of GI symptoms has no association with in-hospital all-cause mortality.
\end{abstract}

Keywords COVID-19 $\cdot$ GI $\cdot$ Diarrhea $\cdot$ Mortality $\cdot$ Outcomes $\cdot$ Coronavirus $\cdot$ SARS-CoV-2

Harsh K. Patel

patelhk.md@gmail.com

1 Department of Internal Medicine, Ochsner Clinic Foundation, 1514 Jefferson Hwy, New Orleans, LA 70121, USA

2 Department of Gastroenterology, Ochsner Clinic Foundation, New Orleans, USA

3 Department of Gastroenterology, Mayo Clinic Arizona, Scottsdale, USA

4 School of Public Health, Louisiana State University Shreveport, Shreveport, USA

5 Ochsner Clinical School, University of Queensland, New Orleans, USA

6 Center for Outcomes and Health Services Research, Ochsner Health System, New Orleans, USA

\section{Introduction}

As of March 15, 2021, more than 124 million people have been infected by the novel coronavirus (SARS-CoV2) and more than 2.5 million patients have died worldwide from the direct or indirect consequences of infection. Although the most common presenting symptoms for these patients are respiratory, with fever and cough being the predominant symptoms [1], there have been many studies on gastrointestinal (GI) manifestations due to COVID-19. This underlies the importance of having high index of suspicion for SARS$\mathrm{CoV}-2$ infection in patients with non-respiratory symptoms $[2,3]$. The angiotensin converting enzyme II (ACE2) receptor that acts as an entry receptor for the SARS-CoV-2 virus $[4,5]$ is highly expressed in the gastrointestinal system in the 
enterocytes in the ileum and colon [6-9] and is the proposed linked to the presence of GI symptoms.

The presence of GI symptoms, including diarrhea, nausea, vomiting and abdominal pain, has been variable in several case series and cohort studies, ranging from 5\% patients in initial reports to as high as 53\% in a recent large nation-wide study $[1,3,10,11]$. There have been studies reporting GI symptoms often precede respiratory symptoms, and in some cases are the only symptoms [12-14]. Given a defined prevalence of GI symptoms in COVID-19, it may be important to understand their association with hospitalization outcomes of COVID-19 patients. We aimed to study the association of GI symptoms at initial hospitalization with clinical outcomes in a large COVID-19 cohort from a single healthcare system at an epicenter in the southern USA.

\section{Materials and Methods}

We retrospectively identified all consecutive patients admitted for COVID-19 infection within our health system between March 1 and August 30, 2020. A COVID-19 diagnosis was based on a qualitative real-time reverse transcriptase polymerase chain reaction (PCR) assay using a nasopharyngeal swab specimen. In-hospital outcomes were assessed until 12 weeks of hospitalization or discharge, whichever occurred later. Patients were excluded if they were age $<18$ years, if they had prior admission with a laboratory confirmed COVID-19 test or if they were never hospitalized after an initial laboratory confirmed COVID-19 test. Ochsner Health, based in New Orleans, is the largest integrated-delivery health system in Louisiana. The institutional review board at Ochsner Health approved this study.

\section{Data Collection}

A system-wide enterprise data warehouse for the Ochsner health system, organized during the initial stage of pandemic, was utilized to extract clinical data from the health system's electronic medical record system (EPIC). We collected the following data/variables for COVID-19 positive hospitalized patients from the data warehouse: demographic characteristics (age, gender, patient-reported race and ethnic group); known comorbid conditions documented through ICD-10 codes (International Classification of Diseases, 10th Revision) linked to ambulatory primary care and specialty encounters (listed below) smoking history and body-mass index (BMI) recorded within the previous 12 months; laboratory data within the previous 30 days; selected outpatient medications; symptoms and diagnosis codes linked to primary care, emergency department (ED), or urgent care encounter during which COVID-19 testing occurred; and vital signs, selected inpatient medications, inpatient procedure codes and diagnoses (listed in appendix), intensive care unit (ICU) transfers or admissions, and discharge outcomes linked to inpatient encounters. Use of the following outpatient medications was collected: (1) Daily use of angiotensin converting enzyme inhibitors (ACEI), or angiotensin-II receptor blockers (ARB) within the previous 12 months, and (2) antibiotic exposure within 14 days prior to admission. Any administration of the following inpatient medications was collected: ACEI/ARB, antibiotics, antivirals, and hydroxychloroquine (HCQ). Use of these medications was collected due to the proposed implications to clinical outcomes in COVID-19 patients.

After initial data extraction from the enterprise data warehouse, manual electronic medical record review was performed by three independent reviewers (EL, MS and HP). Symptom data were manually collected from emergency department assessment and first history and physical admission note. An individual review of the collected de-identified data was independently performed by another reviewer (SP) to identify missing data, and discrepant entries were corrected by re-review of the chart by the responsible reviewer (EL, MS or HP).

The study population was divided into three groups for comparisons: Cohort A: patients with only GI symptoms; Cohort B: patients with GI + non-GI symptoms; and Cohort C: patients with only non-GI symptoms.

\section{Definitions and Outcomes}

Initial hospital presentation corresponded to the first point of contact at an inpatient facility in a patient with a COVID19 PCR positive test and no prior diagnosis of COVID-19. GI symptoms were defined as the presence of one or more of the following four symptoms: diarrhea, nausea, vomiting and abdominal pain. Non-GI symptoms included fever, cough, shortness of breath, sputum production, sore throat, and myalgias. Anorexia and ageusia, being common in other systemic diseases and non-specifically related to the digestive tract, were not considered to be either GI or non-GI symptoms. Tachycardia was defined as heart rate $>100$, hypotension as mean arterial pressure (MAP) $<65 \mathrm{~mm} \mathrm{hg}$ and fever as temperature $>100.3 \mathrm{~F}$. New hemodialysis was defined as patients who required hemodialysis for acute renal failure and who never had dialysis prior to admission. Circulatory shock was defined as hypotension unresponsive to fluid resuscitation and requiring vasopressor administration. Days to ICU was defined as number of days from admission until the step-up to primary critical care service. Days to ventilation was defined as number of days from admission until the requirement for invasive ventilatory support. Length of Stay was defined as duration of hospitalization (days) for patients discharged alive. Per a system-wide protocol antiviral treatment and hydroxychloroquine were only 
administered to patients who had hypoxic respiratory failure, and antibiotics (azithromycin and ceftriaxone) were only administered to patients suspected to have bacterial pneumonia based on initial laboratory (leukocytosis, elevated procalcitonin) and imaging parameters (interstitial infiltrates or focal consolidation on chest $\mathrm{x}$-ray).

The primary outcome was the association of GI symptoms at initial presentation with all-cause in-hospital mortality. Secondary outcomes included: (1) Time-to-event analyses for probability of survival and continued hospitalization, (2) Prevalence of GI symptoms, and (3) Associations of GI symptoms with other clinical manifestations, patient characteristics and clinical events during hospital course (e.g., mechanical ventilation, circulatory shock, need for new hemodialysis, and ICU admission), and 4) Identify predictors of mortality.

\section{Statistical Analysis}

Categorical variables were expressed as numbers and frequencies and compared using Chi-Square test, as outcome variables was separated into 3 groups. Continuous variables were expressed as means with standard deviation (SD) or medians with interquartile range (IQR) depending on the normality of the distribution and compared using an ANOVA test or One-way nonparametric test, respectively. Time-to-event estimate was determined using a Kaplan-Meier estimator. Multivariable logistic regression analysis was used to estimate effects of covariates on mortality. SAS Version 9.4 (SAS Inc., Cary, North Carolina) was used to conduct the analysis. Statistical significance was defined as a $p$ value $<0.05$.

\section{Results}

During the study period, 1672 consecutive patients were admitted with a primary diagnosis of COVID-19 at one of the Ochsner health system hospitals. Patient mean age was $63 \pm 15.8$ years; with $50.4 \%$ females, and this group was predominantly an African American (69.9\%) population. At least one GI symptoms was present on admission in $40.7 \%$. Out of the 1672 patients admitted for COVID-19, $681(40.7 \%)$ patients had at least one GI symptom on initial presentation.

From the 1672 patients admitted for COVID-19, cohort $\boldsymbol{A}$ consisted of 44 patients (2.6\% of entire cohort) presenting with only GI symptoms of a severity that warranted hospitalization, cohort $\boldsymbol{B}$ consisted of 637 patients admitted with both GI and non-GI symptoms, and cohort $\boldsymbol{C}$ consisted of 991 patients admitted with non-GI symptoms.

Among the patients with GI symptoms (cohorts A and B), diarrhea was present in 473 (28.3\%), nausea and/or vomiting in 386 (23.1\%) and abdominal pain in 148 (8.8\%) patients. All three symptoms were present in 2.9 out of every 100 infected patients (Fig. 1).

\section{Comparison of Demographics and Clinical Characteristics (Table 1)}

Gender, race and ethnicity distribution was comparable in all three cohorts. Patients in cohort B were younger and more obese than the other two cohorts $(p<0.001)$. Anorexia and ageusia were significantly higher in cohort B compared to other two cohorts $(p<0.0001)$. Patients in cohort A had higher prevalence of coronary artery disease and chronic kidney disease $(p<0.05)$; patients in cohort $\mathrm{B}$ had higher prevalence of NASH/ NAFLD; patients in cohort $\mathrm{C}$ were more likely to have a pre-existing congestive heart failure or chronic obstructive pulmonary disease $(p<0.05)$. All three cohorts had no differences in the prevalence of diabetes mellitus, hypertension, cirrhosis, solid organ transplant and immunosuppression $(p>0.05)$. Use of ACEI or ARB within 12 months prior to hospitalization or antibiotics within 14 days prior to hospitalization was not significantly different across the cohorts.

Notably, patients in cohort A (only GI symptoms) had a higher prevalence of IBD $(p<0.01)$, and patients in cohort $\mathrm{B}$ (GI and non-GI symptoms) had a higher prevalence of IBS $(p<0.05)$.

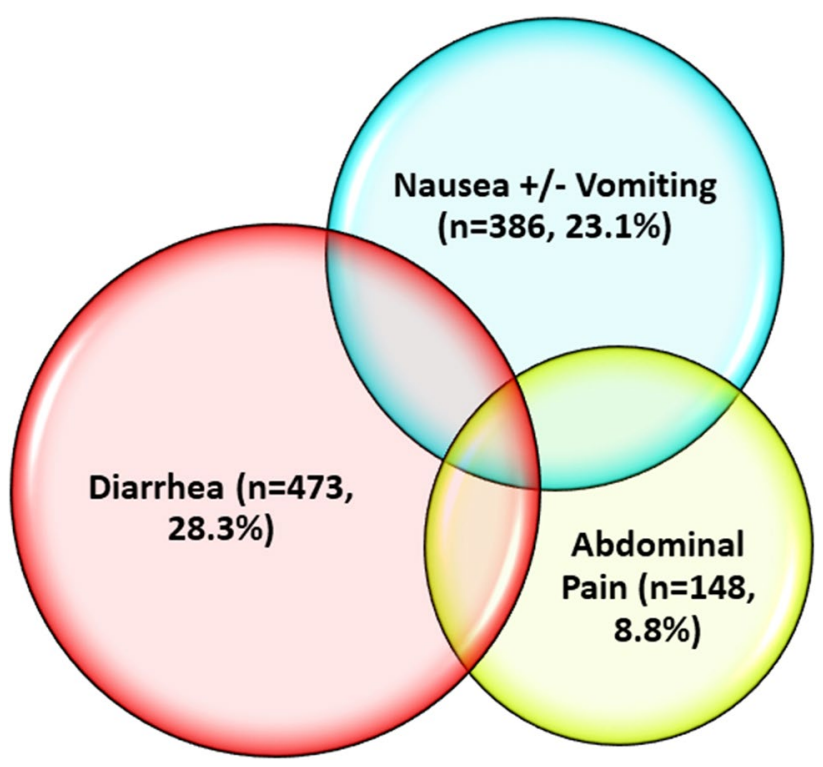

Fig. 1 Presence of GI symptoms in 1672 patients hospitalized with COVID-19 
Table 1 Demographics and Patient Characteristics for all three cohorts

\begin{tabular}{|c|c|c|c|c|c|c|c|}
\hline \multicolumn{3}{|l|}{ Characteristics } & $\begin{array}{l}\text { All patients } \\
(N=1672)\end{array}$ & $\begin{array}{l}\text { Cohort A: Only } \\
\text { GI symptoms } \\
(N=44)\end{array}$ & $\begin{array}{l}\text { Cohort B: } \\
\text { GI+ Non-GI } \\
\text { symptoms } \\
(N=637)\end{array}$ & $\begin{array}{l}\text { Cohort C: Only } \\
\text { non-GI symp- } \\
\text { toms }(N=991)\end{array}$ & $p$ value \\
\hline \multirow[t]{9}{*}{ Demographics } & \multicolumn{2}{|l|}{ Age $($ mean $\pm \mathrm{SD})$} & $63 \pm 15.8$ & $63 \pm 18.7$ & $61 \pm 14.4$ & $65 \pm 16.2$ & $<0.0001$ \\
\hline & Gender N (\%) & Female & $842(50.36)$ & $25(56.82)$ & $321(50.39)$ & $496(50.05)$ & 0.6797 \\
\hline & \multirow[t]{3}{*}{ Race N (\%) } & White & $444(26.56)$ & $11(25)$ & $147(23.08)$ & $286(28.86)$ & \multirow[t]{3}{*}{0.0736} \\
\hline & & Black & $1170(69.98)$ & $33(75)$ & $465(73)$ & $672(67.81)$ & \\
\hline & & Other & $58(3.47)$ & $0(0)$ & $25(3.92)$ & $33(3.33)$ & \\
\hline & Ethnicity N (\%) & $\begin{array}{l}\text { Latino or his- } \\
\text { panic }\end{array}$ & $40(2.39)$ & $0(0)$ & $18(2.83)$ & $22(2.22)$ & 0.4238 \\
\hline & \multicolumn{2}{|c|}{ Body mass index Mean \pm SD } & $32.6 \pm 8.7$ & $29.9 \pm 8.1$ & $33.6 \pm 8.5$ & $32.2 \pm 8.8$ & 0.0007 \\
\hline & \multirow[t]{2}{*}{ Smoking N (\%) } & Never & $1045(62.50)$ & $29(65.91)$ & $406(63.74)$ & $610(61.55)$ & \multirow[t]{2}{*}{0.6029} \\
\hline & & $\begin{array}{l}\text { Former or cur- } \\
\text { rent }\end{array}$ & $627(37.50)$ & $15(34.09)$ & $231(36.26)$ & $381(38.45)$ & \\
\hline \multirow{2}{*}{$\begin{array}{l}\text { Clinical symp- } \\
\text { toms } N(\%)\end{array}$} & \multicolumn{2}{|l|}{ Anorexia } & $690(41.27)$ & $18(40.91)$ & $350(54.95)$ & $322(32.49)$ & $<0.0001$ \\
\hline & \multicolumn{2}{|l|}{ Ageusia } & $66(3.95)$ & $0(0)$ & $44(6.91)$ & $22(2.22)$ & $<0.0001$ \\
\hline \multirow{19}{*}{$\begin{array}{l}\text { Chronic comor- } \\
\text { bid conditions } \\
N(\%)\end{array}$} & \multicolumn{2}{|l|}{ Stroke } & $173(10.35)$ & $7(15.91)$ & $55(8.63)$ & $111(11.2)$ & 0.1188 \\
\hline & \multicolumn{2}{|c|}{ Coronary artery disease } & $261(15.61)$ & $11(25)$ & $84(13.19)$ & $166(16.75)$ & 0.0340 \\
\hline & \multicolumn{2}{|c|}{ Congestive heart failure } & $247(14.77)$ & $6(13.64)$ & $70(10.99)$ & $171(17.26)$ & 0.0023 \\
\hline & \multicolumn{2}{|l|}{ Diabetes mellitus } & $755(45.16)$ & $24(54.55)$ & $286(44.90)$ & $445(44.90)$ & 0.4473 \\
\hline & \multicolumn{2}{|l|}{ Hyperlipidemia } & $738(44.14)$ & $21(47.73)$ & $280(43.96)$ & $437(44.10)$ & 0.8873 \\
\hline & \multicolumn{2}{|l|}{ Atrial fibrillation } & $133(7.95)$ & $4(9.09)$ & $42(6.59)$ & $87(8.78)$ & 0.2712 \\
\hline & \multicolumn{2}{|l|}{ Hypertension } & $1310(78.35)$ & $37(84.09)$ & $495(77.71)$ & $778(78.51)$ & 0.5993 \\
\hline & \multicolumn{2}{|c|}{ Chronic obstructive lung disease } & $199(11.90)$ & $4(9.09)$ & $61(9.58)$ & $134(13.52)$ & 0.0474 \\
\hline & \multicolumn{2}{|c|}{ Asthma } & $179(10.71)$ & $3(6.82)$ & $75(11.77)$ & $101(10.19)$ & 0.4211 \\
\hline & \multicolumn{2}{|c|}{ Chronic kidney disease } & $350(20.93)$ & $15(34.09)$ & $111(17.43)$ & $224(22.60)$ & 0.0041 \\
\hline & \multicolumn{2}{|c|}{ End stage renal disease } & $72(4.31)$ & $3(6.82)$ & $30(4.71)$ & $39(3.94)$ & 0.5337 \\
\hline & \multicolumn{2}{|l|}{ Cirrhosis } & $24(1.44)$ & $0(0)$ & $13(2.04)$ & $11(1.11)$ & 0.2195 \\
\hline & \multicolumn{2}{|c|}{ Chronic viral hepatitis } & $48(2.87)$ & $1(2.27)$ & $18(2.83)$ & $29(2.93)$ & 0.9646 \\
\hline & \multicolumn{2}{|c|}{ Non-alcoholic fatty liver disease } & $45(2.69)$ & $0(0)$ & $25(3.92)$ & $20(2.02)$ & 0.0363 \\
\hline & \multicolumn{2}{|c|}{ HIV infection } & $15(.90)$ & $0(0)$ & $5(.78)$ & $10(1.01)$ & 0.7304 \\
\hline & \multicolumn{2}{|l|}{ Liver transplant } & $27(1.61)$ & $1(2.27)$ & $14(2.20)$ & $12(1.21)$ & 0.2865 \\
\hline & \multicolumn{2}{|c|}{ Other Immunosuppression } & $54(3.23)$ & $0(0)$ & $25(3.92)$ & $29(2.93)$ & 0.2535 \\
\hline & Inflammatory bo & el disease & $34(2.03)$ & $4(9.09)$ & $13(2.04)$ & $17(1.72)$ & 0.0032 \\
\hline & Irritable bowel sy & drome & $16(.96)$ & $0(0)$ & $11(1.73)$ & $5(.50)$ & 0.0378 \\
\hline $\begin{array}{l}\text { Pre-admission } \\
\text { medications } \\
N(\%)\end{array}$ & $\begin{array}{l}\text { Angiotensin conv } \\
\text { inhibitors (ACE } \\
\text { receptor blocke }\end{array}$ & $\begin{array}{l}\text { rting enzyme } \\
\text { /Angiotensin } \\
(\mathrm{ARB})\end{array}$ & $510(30.50)$ & $14(31.82)$ & $182(28.57)$ & 314 (31.69) & 0.4045 \\
\hline & Antibiotics & & $326(19.50)$ & $5(11.36)$ & $136(21.35)$ & 185 (18.67) & 0.1587 \\
\hline
\end{tabular}

Bold values indicate statistically significant results $(p<0.05)$

\section{Comparison of Hospital Treatments and Outcomes (Table 2)}

A smaller fraction of patients in cohort A received treatment in the form of antibiotics and HCQ $(81.8 \%$ and $31.8 \%)$ compared to patients in cohort B (95.9\% and 67.3\%) or cohort C (94.8\% and $64.3 \%)$, respectively. There was no statistically significant difference in the percentage of patients in each cohort requiring ICU admission or the length of stay in the ICU. However, patients in cohort A that required ICU care had a longer interval from initial hospital admission to ICU transfer (4.3 days). The proportion of patients in cohort A requiring endotracheal ventilatory support was less than the other cohorts, but this did not reach statistical significance $(p=0.0758)$. However, the duration from admission to requiring mechanical ventilation was longer in cohort $\mathrm{A}$ compared to the other two cohorts $(p<0.0001)$. Proportionately fewer patients had circulatory shock in cohort A 
Table 2 Clinical Presentation and Clinical Course

\begin{tabular}{|c|c|c|c|c|c|c|}
\hline & & $\begin{array}{l}\text { All patients } \\
(N=1672)\end{array}$ & $\begin{array}{l}\text { Cohort A: Only GI } \\
\text { symptoms }(N=44)\end{array}$ & $\begin{array}{l}\text { Cohort B: GI + Non- } \\
\text { GI symptoms } \\
(N=637)\end{array}$ & $\begin{array}{l}\text { Cohort C: Non- } \\
\text { GI symptoms } \\
(N=991)\end{array}$ & $p$ Value \\
\hline \multirow{3}{*}{$\begin{array}{l}\text { Initial presentation } \\
\text { vital signs } N(\%)\end{array}$} & Tachycardia & $933(55.80)$ & $22(50.0)$ & $358(56.20)$ & $553(55.80)$ & 0.7255 \\
\hline & Hypotension & $223(13.34)$ & $9(20.45)$ & $67(10.52)$ & $147(14.83)$ & 0.0163 \\
\hline & Fever & $756(45.22)$ & $6(13.64)$ & $320(50.24)$ & $430(43.99)$ & $<0.0001$ \\
\hline \multirow{4}{*}{$\begin{array}{l}\text { Hospitalization } \\
\text { medications } N(\%)\end{array}$} & ACEI/ARB & $510(30.50)$ & $14(31.82)$ & $182(28.57)$ & $314(31.69)$ & 0.4045 \\
\hline & Antivirals & $117(7.00)$ & $1(2.27)$ & $47(7.38)$ & $69(6.96)$ & 0.4376 \\
\hline & antibiotics & $1587(94.92)$ & $36(81.82)$ & $611(95.92)$ & $940(94.85)$ & 0.0002 \\
\hline & Hydroxychloroquine & $1080(64.59)$ & $14(31.82)$ & $429(67.35)$ & $637(64.28)$ & $<0.0001$ \\
\hline \multicolumn{2}{|c|}{ New hemodialysis $N(\%)$} & $83(4.96)$ & $1(2.27)$ & $40(6.28)$ & $42(4.24)$ & 0.1275 \\
\hline \multirow[t]{3}{*}{ ICU $N(\%)$} & & $625(37.38)$ & $13(29.55)$ & $231(36.58)$ & $372(38.24)$ & 0.4398 \\
\hline & $\begin{array}{l}\text { Days to ICU } \\
\quad(\text { Mean }, \pm S D)\end{array}$ & $2.39 \pm 3.59$ & $4.31 \pm 5.31$ & $2.88 \pm 4.11$ & $2.02 \pm 3.10$ & 0.0023 \\
\hline & $\begin{array}{l}\text { Number of days in } \\
\text { ICU }(\text { Mean, } \pm S D)\end{array}$ & $9.91 \pm 8.12$ & $7.77 \pm 7.33$ & $9.61 \pm 7.73$ & $10.17 \pm 8.38$ & 0.4487 \\
\hline \multicolumn{2}{|c|}{ Mechanical ventilation N (\%) } & $447(26.73)$ & $6(13.64)$ & $163(25.59)$ & $278(28.05)$ & 0.0758 \\
\hline & $\begin{array}{l}\text { Days to ventilation } \\
\quad(\text { Mean }, \pm S D)\end{array}$ & $2.66 \pm 3.62$ & $7.83 \pm 6.27$ & $3.39 \pm 4.11$ & $2.12 \pm 3.06$ & $<0.0001$ \\
\hline & $\begin{array}{l}\text { Number of days } \\
\text { on ventilation } \\
(\text { Mean, } \pm S D)\end{array}$ & $10.02 \pm 7.37$ & $11.17 \pm 8.52$ & $9.39 \pm 6.49$ & $10.36 \pm 7.82$ & 0.3774 \\
\hline \multicolumn{2}{|l|}{ Shock $N(\%)$} & $460(27.51)$ & $6(13.64)$ & $166(26.06)$ & $288(29.06)$ & 0.0470 \\
\hline \multirow{2}{*}{$\begin{array}{l}\text { Severity of shock } \\
\quad N(\%)\end{array}$} & 1 Vasopressor only & $203(44.13)$ & $1(16.67)$ & 77 (46.39) & $125(43.40)$ & \multirow[t]{2}{*}{0.3263} \\
\hline & $>/=2$ Vasopressors & $257(55.87)$ & $5(83.33)$ & $89(53.61)$ & $163(56.60)$ & \\
\hline \multicolumn{2}{|c|}{ In-hospital all-cause mortality $N(\%)$} & $399(23.86)$ & $9(20.45)$ & $128(20.09)$ & $262(26.44)$ & 0.0118 \\
\hline \multicolumn{2}{|c|}{ Length of stay (Mean, $\pm S D)$} & $10.20 \pm 9.64$ & $7.43 \pm 6.38$ & $9.61 \pm 9.58$ & $10.77 \pm 9.76$ & 0.0229 \\
\hline
\end{tabular}

Bold values indicate statistically significant results $(p<0.05)$

(13.6\%) compared to cohort B (26\%) and cohort C (29\%) $(p<0.05)$. Mortality was significantly higher in cohort $\mathrm{C}$ (26.4\%) compared to cohorts A (20.4\%) and B (20.1\%) $(p<0.05)$. The length of stay was significantly shorter for patients in cohort A (7.4 days) compared to cohort B (9.6 days) or cohort $\mathrm{C}$ (10.8 days).

\section{Time-to-Event Analysis (Fig. 2a and Fig. 2b)}

We performed a Kaplan-Meier analysis for the estimation of survival probability over time for each cohort as well as estimation of probability for continued hospitalization over time for each cohort. There was no statistically significant difference in survival probability between three cohorts at 15 days post-admission $(p=0.3661)$ (Fig. 2a). However, patients in cohort A were least likely to be hospitalized at 15 days post-admission ( $p=0.0014)$ (Fig. 2b).

\section{Predictors of Mortality (Table 3)}

On multivariate logistic regression analysis, the presence or absence of GI symptoms was not a predictor of mortality. Although, the presence of anorexia, gender, administration of ACEI/ARB medications during hospitalization and lower length of stay were all associated with lower odds of death $(p<0.05)$. On the contrary, increasing age, tachycardia on admission day, history of stroke, the presence of CKD, cirrhosis, administration of HCQ, new onset hemodialysis and admission to ICU were all associated with a significantly higher odds of mortality during admission $(p<0.05)$.

\section{Discussion}

This large multicenter retrospective study was conducted by collecting data for in-hospital outcomes on 1672 hospitalized COVID-19 positive patients from the largest nonprofit 
Product Limit Time-To-Event Estimates

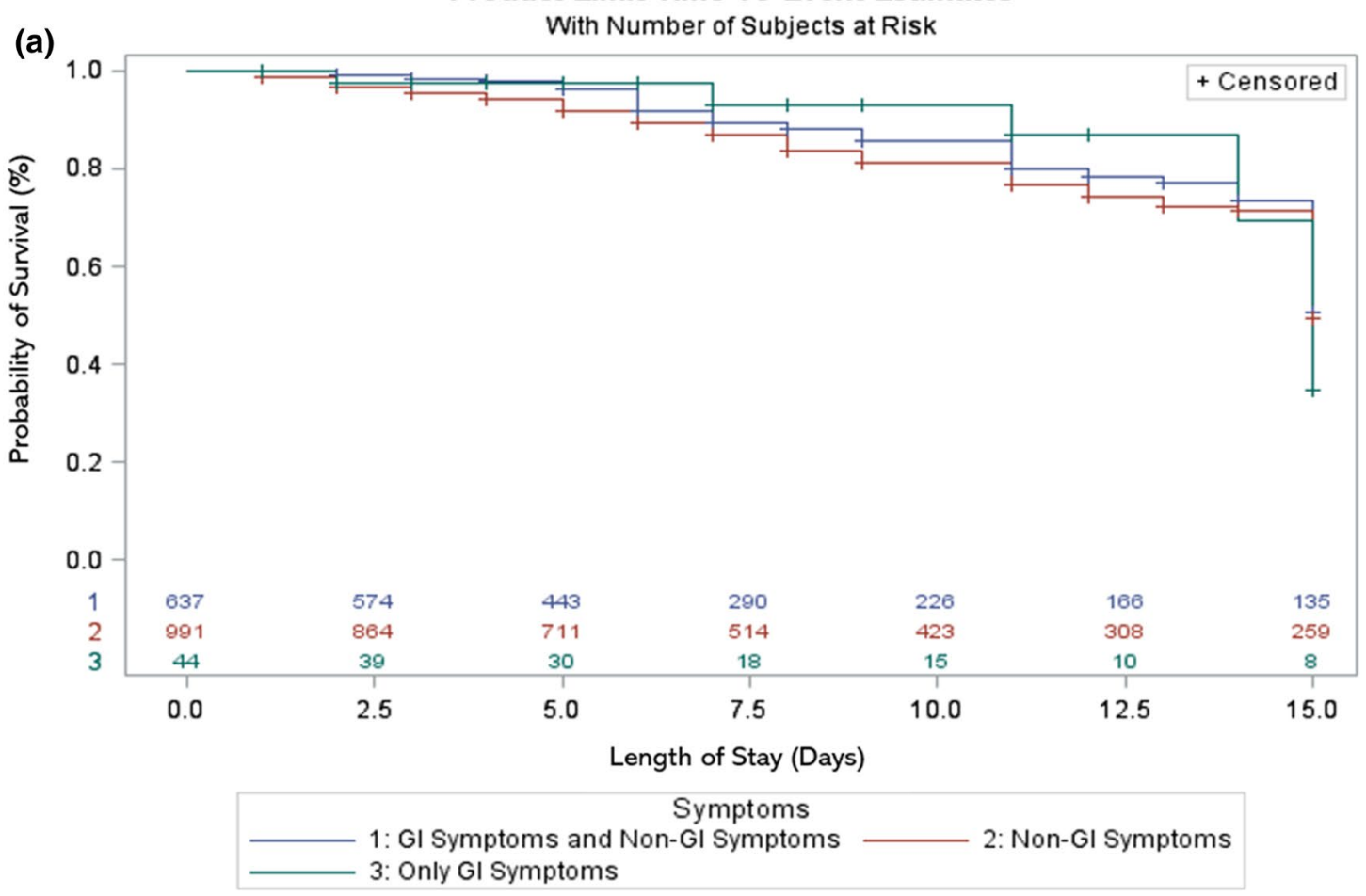

Product Limit Time-To-Event Estimates

(b) With Number of Subjects at Risk

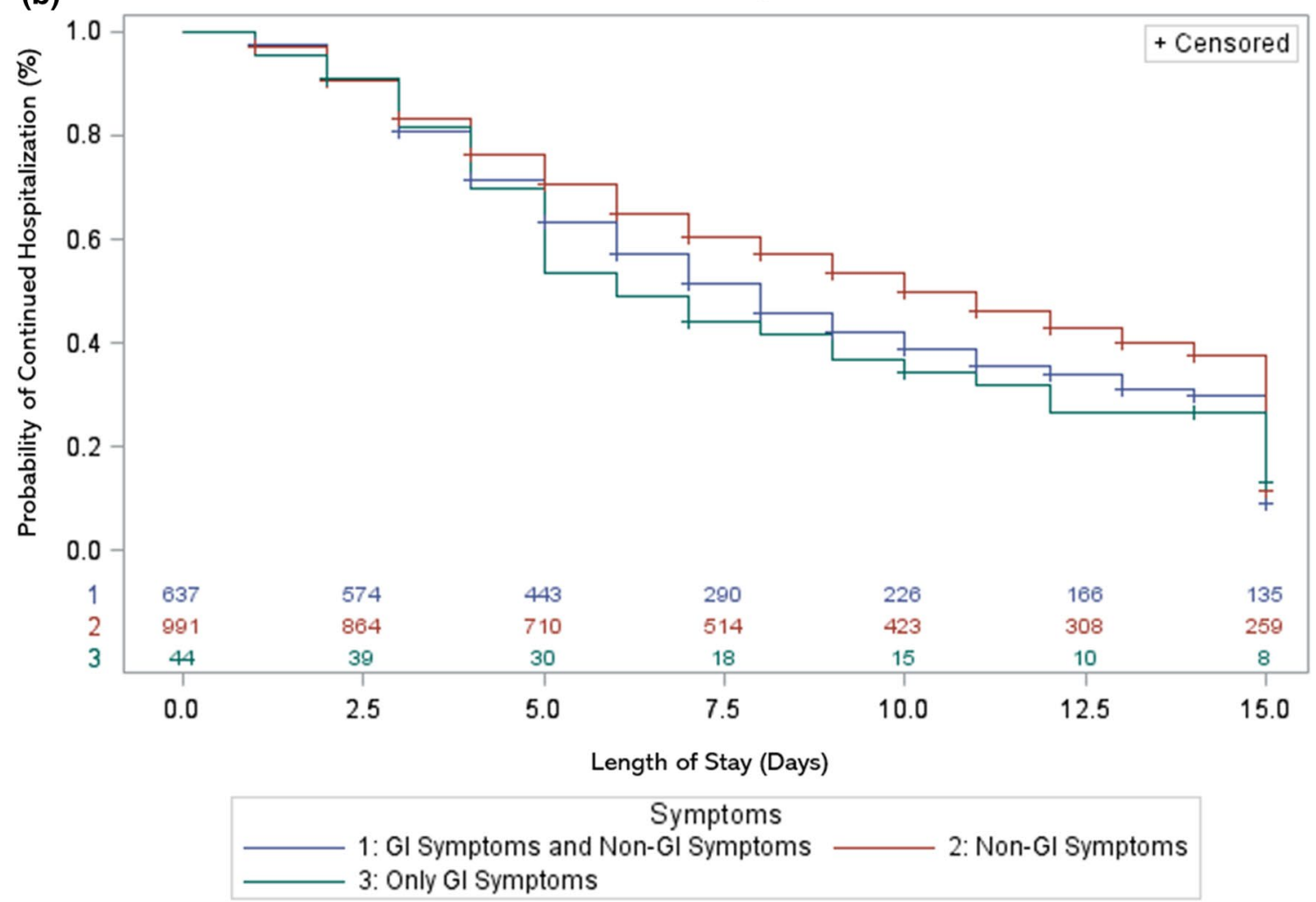

Fig. 2 a Time-to-Event Plot (Kaplan-Meier Analysis): Probability of Survival. b Time-to-Event Plot (Kaplan-Meier Analysis): Probability of Continued Hospitalization 
Table 3 Predictors of Death

\begin{tabular}{lclr}
\hline Multivariate logistic regression model for death \\
\hline Covariates & Odds ratio (OR) & $\begin{array}{l}95 \% \text { confidence } \\
\text { interval }(\mathrm{CI})\end{array}$ & $p$ Value \\
\hline Age $^{\mathrm{a}}$ & & $(2.99,5.40)$ & $<\mathbf{0 . 0 0 0 1}$ \\
Gender $_{\text {Smoking status }}$ & 1.02 & $(0.41,0.74)$ & $<\mathbf{0 . 0 0 0 1}$ \\
Anorexia & 0.55 & $(1.13,2.49)$ & $\mathbf{0 . 0 1 0 8}$ \\
CVA & 0.45 & $(0.31,0.65)$ & $<\mathbf{0 . 0 0 0 1}$ \\
CKD & 1.65 & $(1.07,2.55)$ & $\mathbf{0 . 0 2 3 9}$ \\
Cirrhosis & 2.35 & $(1.33,4.16)$ & $\mathbf{0 . 0 0 3 4}$ \\
Tachycardia & 3.45 & $(1.18,10.05)$ & $\mathbf{0 . 0 2 3 5}$ \\
ACEI ARB & 1.65 & $(1.23,2.23)$ & $\mathbf{0 . 0 0 0 9}$ \\
HCQ & 0.54 & $(0.33,0.88)$ & $\mathbf{0 . 0 1 3 0}$ \\
New hemodialysis & 3.09 & $(1.60,3.38)$ & $<\mathbf{0 . 0 0 0 1}$ \\
ICU & 18.63 & $(1.74,5.48)$ & $\mathbf{0 . 0 0 0 1}$ \\
LOS & 0.95 & $(12.06,28.76)$ & $<\mathbf{0 . 0 0 0 1}$ \\
\end{tabular}

Bold values indicate statistically significant results $(p<0.05)$

${ }^{\text {a }}$ Corresponding to a 15-unit change in Age

academic health care system in Louisiana. With a total of 681 patients with at least one GI symptom, our study shows that $41 \%$ of COVID-19 patients had either diarrhea, nausea \pm vomiting, and/or abdominal pain on initial presentation, and 2.6\% of COVID-19 patients presented with only GI symptom(s). We divided our study population into three cohorts to examine associations of each cohort with admission outcomes and links to pre-admission factors. The presence or absence of GI symptoms was not associated with mortality when effect was adjusted for other pre-admission and admission variables.

Early studies reported a lower prevalence of GI symptoms in hospitalized patients with COVID-19 understandably due to initial under-recognition of GI symptoms as a spectrum of phenotypic presentation in COVID-19. Higher prevalence reported in later studies may be somewhat inflated due to attributing anorexia as a specific GI symptom [14]. In our study, diarrhea was the most common GI symptom on presentation followed by nausea, vomiting and abdominal pain. We did not classify anorexia and ageusia as GI symptom at initial presentation, but did find that patients have significantly higher odds of having at least one GI symptom when they have either anorexia or ageusia. If we include anorexia as one of the GI symptoms on presentation, the prevalence of GI symptoms would increase to $60 \%$ in our study.

We identified that younger patients had a higher prevalence of GI symptoms at initial presentation. This is similar to findings from a recent study [11]. Another recent study reported that expression of ACE2 on intestinal enterocytes cells and colonic epithelial cells increases with increasing age which could explain the lower prevalence of gastrointestinal symptoms [15]. How this applies to prevalence of GI symptoms in the younger population with a lower ACE-2 expression remains to be understood.

Tan et al. reported that inpatient use of ACEI or ARB medications in COVID-19 patients was associated with lower rate of GI symptoms during hospitalization and was limited by a very small sample size $(n=31)$ [16]. However, no prior study has assessed the relation between development of GI symptoms and the use of these medication prior to hospitalization. We aimed to address the influence of ACEI and ARB medications by defining the presence of GI symptoms at initial presentation, by assessing the outpatient use of those medications, and by including a larger number of patients taking either medication $(n=510)$. We report no difference in prevalence of GI symptoms in patients who were taking ACEI or ARB medication prior to admission compared to those who were not. Despite these medications being continued inpatient in similar proportions in the three cohorts, inpatient use of ACEI/ARB in our study was associated with improved mortality which has been reported in some recent studies as well $[17,18]$.

In our study, patients with only GI symptoms had a lower mean BMI compared to other patients. Prior studies have assessed the difference in severity outcomes of obese patients with COVID-19 infection and found obesity to be significantly associated with critical infection $[19,20]$, but the association of obesity with the presence of GI symptoms has been not been studied. Our results provide an interesting association between lower BMI and a higher prevalence of only GI symptoms at initial presentation. Similarly, we also identified that patients with NAFLD were significantly more likely to present with GI and non-GI symptoms. In fact, none of the NAFLD patients presented with only GI symptoms. NAFLD has long been known to be a multisystem disease and is also significantly related to obesity [21]. The difference in prevalence of symptoms in patients with NAFLD is in line with the BMI differences in the three cohorts.

Patients with IBS had higher prevalence of GI symptoms along with non-GI symptoms in our study. This could be due to the fact that IBS is significantly associated with anxiety in patients and thereby could manifest as shortness of breath on initial presentation [22]. Additionally, none of the IBS patients presented with only GI symptoms. We suspect that the patients with diagnoses of IBS already had evaluations with a specialist and understanding of GI symptomatology related to IBS, which may in turn have reduced thresholds for clinical evaluations unless other non-GI symptoms were also present. Patients with IBD, on the contrary, were significantly more likely to present with only GI symptoms upon initial presentation. Whether this truly reflects a direct association between IBD and increase in COVID-19 related GI symptoms versus just increased patient concern for a disease flare with coincidental COVID-19 infection is unknown. 
Some prior studies have demonstrated significantly poorer clinical outcomes in hospitalized patients presenting with GI symptoms compared to those without GI symptoms. These clinical outcomes included disease severity, critical infection requiring ICU admission, requirement of mechanical ventilation, increased incidence of liver injury, and mortality [2, 14, 23]. However, there have been other studies that reported no correlation between the presence of GI symptoms and these outcome measures [10, 24, 25].

Some studies have reported improved mortality in COVID-19 patients with GI symptoms [11, 26, 27]. However, these studies were limited due to either only including hospitalized patients with mild disease severity, only including the outpatient population (likely only having mild disease severity), or not analyzing outcomes in patients presenting with only GI symptoms. We believe the data from our large cohort adds value to the current literature, addresses the variability in the outcomes reported in COVID-19 inpatients with GI symptoms, and reports outcomes with respect to patients presenting with only GI symptoms.

Although there was no difference among patients presenting with only GI symptoms to require transfer to ICU for critical illness, there was a significant longer duration before transfer to the ICU in this group. This is likely explained by a delayed development of respiratory compromise in a subset of these patients who initially only presented with GI symptoms. However, with respect to another possible correlate of disease severity, circulatory shock developed in significantly fewer patients presenting with only GI symptoms. We also report that the presence of GI symptoms, independent of the presence of any non-GI symptom at admission was associated with a lower mortality compared to the presence of only non-GI symptoms $(p=0.018)$. This did not impact the survival prognosis at 15 days on a Kaplan-Meier analysis due to a high number of patients being censored (discharged alive) within 2 weeks. To partially account for this, we also performed a time-to-event analysis to estimate the probability of continued hospitalization at 15 days. Patients presenting with only GI symptoms had the least probability of continued hospitalization at 15 days compared to patients with respiratory symptoms at initial presentation.

A lot of prior work addressing this question did not clearly evaluate confounding effects of pre-admission and admission factors, and conclusions could have borne out of over or under-estimation of GI symptoms as predictors of mortality. After consideration of the inter-group differences in characteristics and management during admission in our study, i.e., on a multivariable regression analysis, GI symptoms on presentation did not predict mortality in these patients. Our study adds to the evidence on this important clinical prognostication question about outcomes of COVID19 in hospitalized patients presenting with GI symptoms, where considerable heterogeneity exists.
Our study's major strengths are the large cohort size and the availability of discharge outcomes in all the patients. We gathered data on a larger number of patients with GI manifestations $(n=681)$ compared to numbers reported from most prior studies, even after excluding anorexia as a GI symptom. We also report important associations between GI symptoms on presentation and hospital outcomes of mortality and correlates of infection severity (e.g., ICU admission, rates of mechanical ventilation, shock, length of stay).

Our study does have limitations. This was a non-blinded retrospective study that was based on data gathered through medical record review and thus, has a potential to introducing bias. To minimize the errors, more than one reviewer independently verified the accuracy of the collected data. Ambulatory COVID-19 positive patients with and without GI symptoms that did not require hospitalization are not included. Our data lacked the information on symptom severity which may have impacted outcomes. We did not collect data on pre-admission or admission use of proton pump inhibitors or $\mathrm{H} 2$ receptor blockers, but a recent study has found no effect of use of these medication on hospitalization outcomes [28]. Longitudinal follow-up of these patients $>3$ months for prognosis and relation to GI symptoms is beyond the scope of our study. Nevertheless, our study provides clinically relevant information on the overall burden of digestive manifestations and their association with mortality and other important COVID-19-related hospitalization outcomes.

\section{Conclusion}

In summary, among a hospitalized COVID-19 positive Southern US population, $41 \%$ patients presented with either diarrhea, nausea, vomiting or abdominal pain initially. Even though it appeared that the presence of GI symptoms at presentation correlated with a reduced mortality rate and shorter hospitalization and appeared to be a positive prognosticator, but the correlation with mortality was eventually not consistent after adjusting for various pre-admission and admission factors between cohorts. There is significant heterogeneity in outcomes related to GI symptoms from both small and large prior studies. Our large retrospective study, with a multivariable analysis extensively adjusting for variables, adds substantial evidence to the existing body of the literature in COVID-19. 


\section{Appendix}

\section{ICD- 10 Diagnostic Codes}

For Pre-admission Chronic Comorbid Conditions.

CVA (I63.9), CAD (I25.1; I25.10; I25.110; I25.118; I25.119) CHF (I50; I50.2; I50.20; I50.21; I50.22; I50.23; I50.3; I50.30; I50.31; I50.32; I50.33;I50.4; I50.40; I50.41;I50.42; I50.43; I50.9; I50.810; I42.0; I42.1; I42.5; I42.8; I25.5), DM (E10.9; E10.21; E10.22; E10.29; E10.39; E10.319; E10.49; E10.40; E10.42; E10.43; E10.51; E10.52; E10.59; E10.69; E11.9; E11.21; E11.22; E11.29; E11.39; E11.319; E11.49; E11.40; E11.42; E11.43; E11.51; E11.52; E11.59; E11.69), HTN (I10; I15; I15.8; I15.9), COPD (J44.1; J44.9; J41.0; J41.1; J42; J43.9; J43.1; J43.2) Asthma (J45.901; J45.902; J45.909; J45.30; J45.31; J45.32; J45.40; J45.41; J45.42; J45.50; J45.51; J45.52), CKD (N18.1; N18.2; N18.3; N18.4; N18.5), ESRD (N18.6), Cirrhosis (K70.30; K70.31; K74.60, K74.61, K74.3; K74.4; K74.5; K74.69), Alcoholic Liver disease (K70.0; K70.10; K70.11), NASH/NAFLD (K75.81; K76.0), Hepatitis (B15.9; B19.10; B19.20; B19.9; B18.2; K75.4), HIV (B20; Z21), Transplant (Z94.0; Z94.1; Z94.2; Z94.4; Z94.81; Z94.83; Z94.84 Z94.9), Immunosuppression (Z79.52; Z79.899; D89.9; Z92.25); IBD (K51.20; K51.30; K51.90; K50.00; K50.10; K50.80; K50.90; K52.9) IBS (K58.0; K58.1; K58.2; K58.8; K58.9), Chronic Pancreatitis (K86.0; K86.1)

\section{ICD-10 Procedure and Diagnostic Codes}

For inpatient procedures and diagnoses J10 through J18, N17, K72, I42, J96, J80, E11.10, and E87.2

*Data on laboratories and multivariate analysis for predictors of GI symptoms are available at request

\section{Acknowledgments No funding source to disclose.}

Author's contribution Conceptualization was contributed by HKP, JNS; Data curation was contributed by HKP, JHB, SCP, MS, ELC; Formal analysis was contributed by HKP, RK; Methodology was contributed by HKP, VTC, JNS; Project administration was contributed by HKP, SCP; Supervision was contributed by AR, JNS; Writing (original draft) was contributed by HKP, VTC; Writing (critical review \& editing) was contributed by all authors; Final approval was contributed by all authors.

\section{Declarations}

Conflict of interest The following authors declare that they have no conflict of interest: Harsh K. Patel, Rosemary Kovacic, Viveksandeep Thoguluva Chandrasekar, Sneha C. Patel, Munraj Singh, Elise Le
Cam, Jeffrey H. Burton, Janak N. Shah. Consultant for Rebiotix: Arnab Ray.

\section{References}

1. Guan W-J, Ni Z-Y, Hu Y, et al. Clinical Characteristics of Coronavirus Disease 2019 in China. N Engl J Med. 2020:1-13. doi:https:// doi.org/10.1056/NEJMoa2002032

2. Cheung KS, Hung IFN, Chan PPY, et al. Gastrointestinal Manifestations of SARS-CoV-2 Infection and Virus Load in Fecal Samples From a Hong Kong Cohort: Systematic Review and Metaanalysis. Gastroenterology. 2020:81-95. doi:https://doi.org/10. 1053/j.gastro.2020.03.065

3. Parasa S, Desai M, Thoguluva Chandrasekar V et al. Prevalence of Gastrointestinal Symptoms and Fecal Viral Shedding in Patients With Coronavirus Disease 2019: A Systematic Review and Metaanalysis. JAMA Netw open. 2020;3:e2011335-e2011335. https:// doi.org/10.1001/jamanetworkopen.2020.11335.

4. Hoffmann M, Kleine-Weber H, Schroeder S et al. SARS-CoV-2 Cell Entry Depends on ACE2 and TMPRSS2 and Is Blocked by a Clinically Proven Protease Inhibitor. Cell. 2020;181:271-280. e8. https://doi.org/10.1016/j.cell.2020.02.052.

5. Shang J, Ye G, Shi K et al. Structural basis of receptor recognition by SARS-CoV-2. Nature. 2020;581:221-224. https://doi. org/10.1038/s41586-020-2179-y.

6. Xu H, Zhong L, Deng J et al. High expression of ACE2 receptor of 2019-nCoV on the epithelial cells of oral mucosa. Int J Oral Sci. 2020;12:8. https://doi.org/10.1038/s41368-020-0074-x.

7. Li M-Y, Li L, Zhang Y, Wang X-S. Expression of the SARSCoV-2 cell receptor gene ACE2 in a wide variety of human tissues. Infect Dis Poverty. 2020;9:45. https://doi.org/10.1186/ s40249-020-00662-x.

8. Puelles VG, Lütgehetmann M, Lindenmeyer MT et al. Multiorgan and Renal Tropism of SARS-CoV-2. N Engl J Med. 2020. https://doi.org/10.1056/NEJMc2011400.

9. Zhang H, Kang Z, Gong H, et al. Digestive system is a potential route of COVID-19: an analysis of single-cell coexpression pattern of key proteins in viral entry process. Gut. 2020;69(6):1010 LP - 1018. doi:https://doi.org/10.1136/gutjnl-2020-320953

10. Elmunzer BJ, Spitzer RL, Foster LD, et al. Digestive Manifestations in Patients Hospitalized with COVID-19. medRxiv. 2020. doi:https://doi.org/10.1101/2020.07.07.20143024

11. Laszkowska M, Faye AS, Kim J et al. Disease Course and Outcomes of COVID-19 Among Hospitalized Patients with Gastrointestinal Manifestations. Clin Gastroenterol Hepatol. 2020. https://doi.org/10.1016/j.cgh.2020.09.037.

12. Wang D, Hu B, Hu C et al. Clinical Characteristics of 138 Hospitalized Patients With 2019 Novel Coronavirus-Infected Pneumonia in Wuhan, China. JAMA. 2020;323:1061-1069. https:// doi.org/10.1001/jama.2020.1585.

13. Han C, Duan C, Zhang S, Spiegel B, Shi H, Wang W. Digestive Symptoms in COVID-19 Patients With Mild Disease Severity : Clinical Presentation, Stool Viral RNA Testing, and Outcomes. 2020:1-8.

14. Pan L, Mu M, Ren HG, Yang P. Clinical characteristics of COVID-19 patients with digestive symptoms in Hubei, China : a descriptive, cross-sectional, multicenter study. Am J Gastroenterol. 2020.

15. Vuille RN, Liechty KW, Verrey F, Guglielmetti LC. SARS $\mathrm{CoV}$ - 2 receptor ACE2 gene expression in small intestine correlates with age. Amino Acids. 2020;(July):4-6. doi:https://doi. org/10.1007/s00726-020-02870-z 
16. Tan N, Qiu Y, Xing X, Ghosh S, Chen M, Mao R. Associations between Angiotensin Converting Enzyme Inhibitors and Angiotensin II Receptor Blocker Use, Gastrointestinal Symptoms, and Mortality among Patients with COVID-19. Gastroenterology. 2020. https://doi.org/10.1053/j.gastro.2020.05.034.

17. Zhang $\mathrm{P}$, Zhu L, Cai J et al. Association of Inpatient Use of Angiotensin-Converting Enzyme Inhibitors and Angiotensin II Receptor Blockers With Mortality Among Patients With Hypertension Hospitalized With COVID-19. Circ Res. 2020;126:1671-1681. https://doi.org/10.1161/CIRCRESAHA. 120.317134 .

18. Bean DM, Kraljevic Z, Searle T et al. Angiotensin-converting enzyme inhibitors and angiotensin II receptor blockers are not associated with severe COVID-19 infection in a multi-site UK acute hospital trust. Eur J Heart Fail. 2020;22:967-974. https:// doi.org/10.1002/ejhf.1924.

19. Petrilli CM, Jones SA, Yang J, et al. Factors associated with hospital admission and critical illness among 5279 people with coronavirus disease 2019 in New York City: prospective cohort study. BMJ. 2020;369. doi:https://doi.org/10.1136/bmj.m1966

20. Lighter J, Phillips M, Hochman S et al. Obesity in Patients Younger Than 60 Years Is a Risk Factor for COVID-19 Hospital Admission. Clin Infect Dis an Off Publ Infect Dis Soc Am. 2020;71:896-897. https://doi.org/10.1093/cid/ciaa415.

21. Byrne CD, Targher G. NAFLD: a multisystem disease. J Hepatol. 2015;62:S47-64. https://doi.org/10.1016/j.jhep.2014.12.012.

22. Lydiard RB. Irritable bowel syndrome, anxiety, and depression: what are the links? J Clin Psychiatry. 2001;62:37-38.

23. Jin X, Lian J-S, Hu J-H, et al. Epidemiological, clinical and virological characteristics of 74 cases of coronavirus-infected disease 2019 (COVID-19) with gastrointestinal symptoms. Gut. 2020:1-8. doi:https://doi.org/10.1136/gutjnl-2020-320926
24. Redd WD, Zhou JC, Hathorn KE, et al. Prevalence and Characteristics of Gastrointestinal Symptoms in Patients With Severe Acute Respiratory Syndrome Coronavirus 2 Infection in the United States: A Multicenter Cohort Study. Gastroenterology. 2020;(July):1-3. doi:https://doi.org/10.1053/j.gastro.2020.04.045

25. Remes-Troche JM, Ramos-de-la-Medina A, Manríquez-Reyes M, Martínez-Pérez-Maldonado L, Lara EL, Solís-González MA. Initial Gastrointestinal Manifestations in Patients with SARS-CoV-2 in 112 patients from Veracruz (Southeastern Mexico). Gastroenterology. 2020;S0016-5085:34708-34709. https://doi.org/10. 1053/j.gastro.2020.05.055.

26. Nobel YR, Phipps M, Zucker J et al. Gastrointestinal Symptoms and COVID-19: Case-Control Study from the United States. Gastroenterology. 2020. https://doi.org/10.1053/j.gastro.2020.04.017.

27. Han C, Duan C, Zhang S, Spiegel B, Shi H. Digestive Symptoms in COVID-19 Patients with Mild Disease Severity : Clinical Presentation, Stool Viral RNA Testing, and Outcomes. Am J Gastroenterol.

28. Elmunzer BJ, Wolf BJ, Scheiman JM, Tierney WM, Taylor JR. Association Between Preadmission Acid Suppressive Medication Exposure and Severity of Illness in Patients Hospitalized With COVID-19. Gastroenterology. 2021;160:1417-1422.e14. https:// doi.org/10.1053/j.gastro.2020.11.007.

Publisher's Note Springer Nature remains neutral with regard to jurisdictional claims in published maps and institutional affiliations. 